\title{
Pyroelectric Composite Film for X-ray Intensity Detection
}

\author{
Walter Katsumi Sakamoto $^{\text {**, Giuliano Pierre Estevam }}{ }^{\mathrm{b}}$, Aparecido Augusto de Carvalho ${ }^{\mathrm{c}}$, \\ Wesley Pontes ${ }^{\mathrm{b}}$, Mauro Henrique de Paula ${ }^{\mathrm{d}}$ (in memoriam)
}

\author{
${ }^{\mathrm{a}}$ Grupo de Polímeros, Departamento de Fisica e Química, Faculdade de Engenharia, \\ Universidade Estadual Paulista - UNESP, Campus de Ilha Solteira, \\ Av. Brasil, 56, CEP 15385-000, Ilha Solteira, SP, Brazil \\ ${ }^{\mathrm{b}}$ Faculdade de Tecnologia - FATEC - Araçatuba, Av. Pestes Maia, 1764, \\ CEP 16052-045, Araçatuba, SP, Brazil

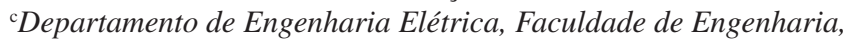 \\ Universidade Estadual Paulista - UNESP, Campus de Ilha Solteira, \\ Av. Brasil, 56, CEP 15385-000, Ilha Solteira, SP, Brazil \\ ${ }^{\mathrm{d} D e p a r t a m e n t o ~ d e ~ F i ́ s i c a, ~ U n i v e r s i d a d e ~ F e d e r a l ~ d e ~ M a t o ~ G r o s s o ~ d o ~ S u l ~-~ U F M S, ~}$ \\ CP 549, CEP 79070-900, Campo Grande, MS, Brazil \\ Received: March 18, 2011; Revised: February 3, 2012
}

\begin{abstract}
Composite material obtained with modified lead titanate (Pz34) ferroelectric ceramic and polyether-ether-ketone (PEEK) polymer matrix was used as sensitive component to measure X-ray intensity in a novel detection system. The sensing element works as a thermal transducer, converting a non-quantified thermal flux into an output measurable quantity of electrical voltage. The samples were obtained up to 60 vol.\% of ceramic, by hot pressing the mixture of Pz34 and PEEK powders at $368^{\circ} \mathrm{C}$ and applying $12 \mathrm{MPa}$ pressure for 2.0 hours. The sensor response varies from 2.70 to $0.80 \mathrm{~V}$ in the energy fluence rate range of 6.30 to $37.20 \mathrm{~W} \cdot \mathrm{m}^{-2}$. The absorbed incident energy was analyzed as a function of the ionizing energy. Furthermore, by measuring the pyroelectric activity of the composite film it was observed that there is no degradation of the sensor after the irradiation.
\end{abstract}

Keywords: composite, pyroelectricity, $X$-ray radiation sensor

\section{Introduction}

Ferroelectric polymers, ferroelectric ceramics and related materials have been used as thermal transducer due to their pyroelectric activity. Radiant energy, both ionizing (X-ray, protons, neutrons) and non-ionizing (infrared, microwaves, UV) are monitored by these sensors ${ }^{1-4}$. In the medical area, cancer has been the worst health problem worldwide and radiation therapy is one of the most effective methods in cancer treatment. Hence, the dose radiation must be precisely and accurately delivered to avoid side effects as well as recurrence of the disease.

According to Cameron ${ }^{5}$ small exposure to radiation can reduce the risk of disease, but it is well known that high dose of radiation may damage healthy tissues, hence becoming very tragic. Determining the values of various dose quantities for use in research, in therapy and in a dose limiting system for protection is the final purpose of radiation dosimetry ${ }^{6}$.

Among the commonly used dosimeters in radiation therapy, the ionization chamber is one of the most accurate and is considered as the gold standard for radiation dosimetry. However, there are some limitations related to the best spatial resolution and the signal-to-noise ratio ${ }^{7}$. Furthermore, ionization chambers are expensive, which is also a limitation.

Radiographic films and thermo luminescent materials have also been used for dosimetry of ionization radiation

*e-mail: sakamoto@dfq.feis.unesp.br but these dosimeters do not provide a direct reading of the dose and the useful information about the dose only can be obtained after tedious set up procedures and further treatment after irradiation ${ }^{8}$.

Semiconductor silicon diodes and solid-state scintillation detectors are widely used in dosimeters and in technological equipment for medical diagnosis. However the small area of the photosensitive surface of the photodiode is one of the major factors limiting the use of these detectors?.

Recently, De Paula et al. ${ }^{10}$ proposed a microcontrolled pyroelectric instrument for measuring X-ray intensity as an alternative to the expensive ionization chamber. The authors have used lithium tantalate $(\mathrm{LiTaO} 3)$ and lithium niobate ( $\mathrm{LiNb5O3)}$ ferroelectric crystals as detectors for monitoring $\mathrm{X}$-ray intensity in the mammography range.

Pyroelectric detectors offer an advantage of room temperature operation and wide spectral response. Material such as triglycine sulphate (TGS), lithium tantalate, and lead zirconate titanate (PZT) have been used as pyroelectric elements in many devices. In the present paper, a ferroelectric ceramic/polymer composite is presented as an alternative pyroelectric detector to measure X-ray intensity in the orthovoltage range $(120-300 \mathrm{kVp})$. The composite material combines good properties of each single material, offering some advantages in comparison with single crystalline materials or ferroelectric perovskite ceramics. 


\section{Experimental}

\subsection{Polymer matrix}

Polyether-ether-ketone (PEEK) is an aromatic polyether. Because of characteristics such as mechanical strength, glass transition temperature around $143{ }^{\circ} \mathrm{C}$, resistance to a large range of chemicals, $\mathrm{PEEK}$ is considered as high temperature engineering thermoplastic ${ }^{11,12}$. On the dielectric side, there is no relaxation in the frequency range of 1.0 to $105 \mathrm{~Hz}$ and the relative permittivity is around $3.5^{13}$. PEEK powder with mean particle size of $80 \mu \mathrm{m}$ was purchased from Goodfellow.

\subsection{Ferroelectric ceramic}

Modified lead titanate (Pz34) powder, purchased from Ferroperm Piezoceramics A/S, exhibit large electromechanical anisotropy and low dielectric constant. Table 1 shows some properties of $\mathrm{Pz} 34^{14}$. The mean grain size of the ceramic is $2 \mu \mathrm{m}$.

\subsection{Composite preparation}

Pz34/PEEK composite films were obtained by pressing the mixture of both powders at $368{ }^{\circ} \mathrm{C}$ for 2 horas. Samples in the thickness range of 150-300 $\mu \mathrm{m}$ were obtained after applying a pressure of $12 \mathrm{MPa}$. The ceramic volume fraction used to prepare the composite film was 50 and 60 vol.\%. The amount of Pz34 and PEEK was calculated using the Equation 1.

$$
M_{c}=M_{p} \frac{\rho_{c}}{\rho_{p}}\left(\frac{\Phi_{c}}{1-\Phi_{c}}\right)
$$

where, $M$ is the mass, $\rho$ is the density and the subscripts $p$ and $\mathrm{c}$ are related with polymer and ceramic, respectively. $\Phi c$ denotes the volume fraction of ceramic.

\subsection{Composite characterization}

Circular aluminum electrodes with $10 \mathrm{~mm}$ of diameter were vacuum evaporated onto both sides of the composite film for electrical contact. The samples were poled with appropriate electric field, and to avoid breakdown the polarization was performed in silicone oil bath with controlled temperature.

Longitudinal piezoelectric coefficient $d 33$ was measured using a Pennebaker model 8000 d33 Piezo Tester (APC) at $60 \mathrm{~Hz}$. The piezoelectric activity of the composite films was studied as a function of poling electric field. The pyroelectric coefficient $(\mathrm{p}(\mathrm{t}))$ was also determined using the direct method of measuring the pyroelectric current ${ }^{15}$.

\subsection{Radiation detection system}

Figure 1 shows the pyroelectric chamber constructed to measure X-ray intensity. The prototype instrument consists of a pyroelectric sensor (composite), a low-noise high sensitivity current-to-voltage converter, a microcontroller and a digital display.

Table 1. Values of density, piezoelectric coefficient, dielectric constant and Curie temperature.

\begin{tabular}{ccccc}
\hline Material & $\rho\left(\mathbf{g} . c m^{-3}\right)$ & $\mathbf{d}_{\mathbf{3 3}}(\mathbf{p C} / \mathbf{N})$ & $\varepsilon_{\mathbf{r}}(\mathbf{1} \mathbf{k H z})$ & $\mathbf{T}_{\mathbf{c}}\left({ }^{\circ} \mathbf{C}\right)$ \\
\hline Pz34 & 7.55 & 50 & 210 & 400 \\
\hline
\end{tabular}

The main component of the converter is an operational amplifier OPA 111 (Burr-Brown), which has very high input impedance $\left(10^{14} \Omega / / 3 \mathrm{pF}\right)$, low input bias $(0.8 \mathrm{pA})$ and offset currents $(0.5 \mathrm{pA})$. In order to minimize noise from external sources, the current-to-voltage converter was placed inside the pyroelectric chamber, which is a grounded aluminum enclosure. Using guard-rings around the input pins of the operational amplifier minimized leakage currents across the fiberglass printed circuit board. The guard-ring acts also as shield against noise ${ }^{16}$.

To protect the electronic circuit from radiation, it was placed inside a compartment in the pyroelectric chamber that was shielded with a $7 \mathrm{~mm}$ thick alloy (cerobend 70 ) composed of $50 \%$ of bismuth, $27 \%$ of lead, $13 \%$ of tin and $10 \%$ of cadmium. The signals in the output of the converter are noise filtered and then fed into the micro-controller acquisition section. The microcontroller (Microchip PIC 16F777) sends the digital converted analogical signal to a display. Figure 2 shows the lateral view of the pyroelectric chamber and a block diagram of the radiation detector system.

The irradiations were carried out using orthovoltage X-ray equipment (Siemens Stabilipan II). The characteristics of the instrument are described in Table 2.

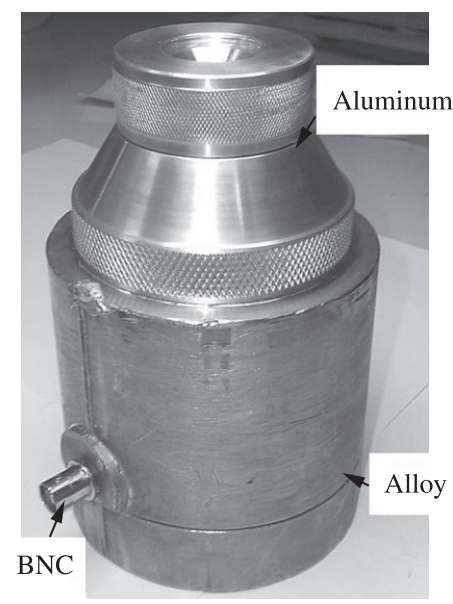

(a)

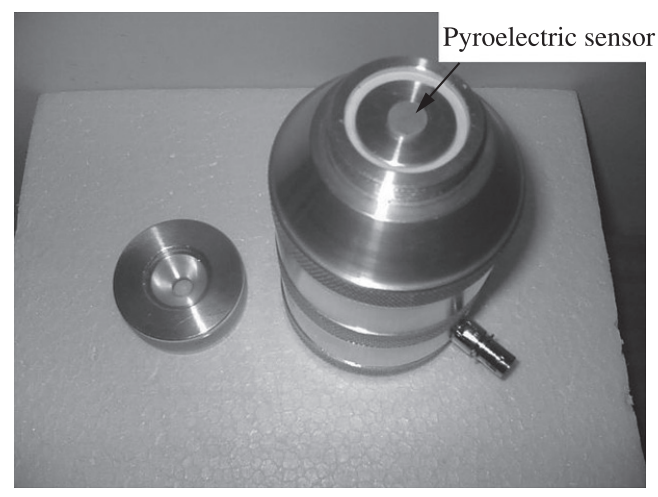

(b)

Figure 1. Photograph of the pyroelectric chamber a) closed; and b) showing the composite sensor. 


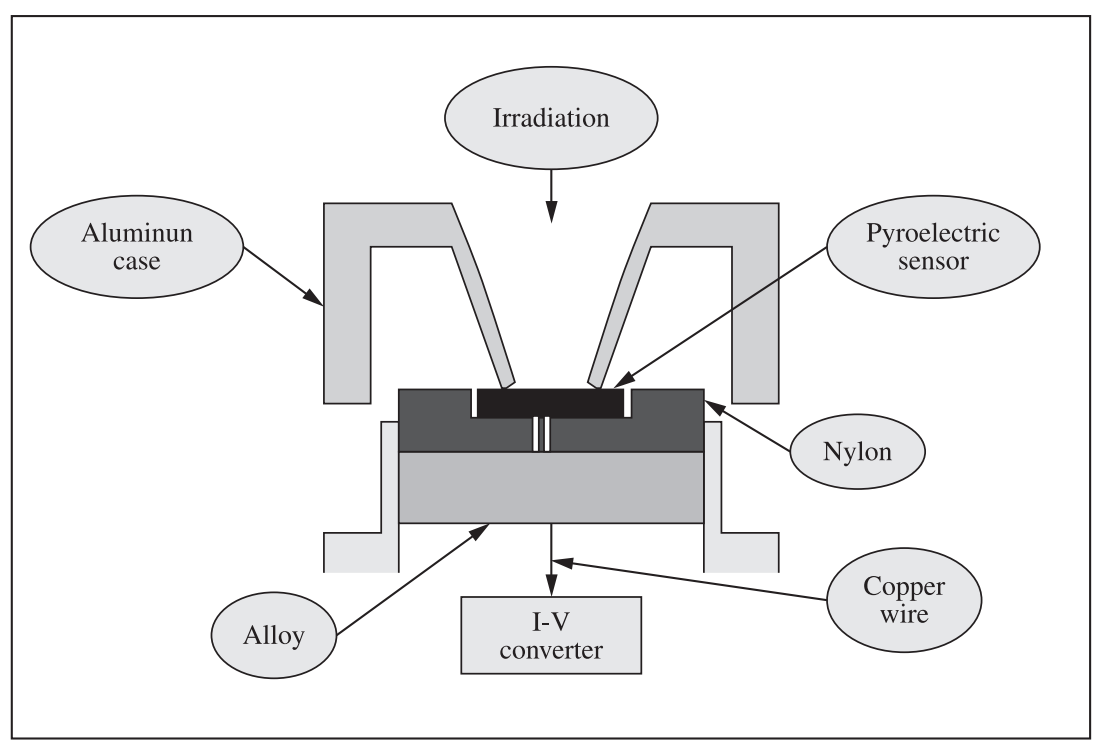

(a)

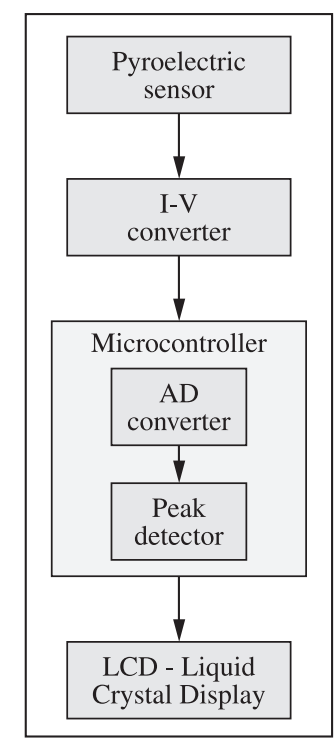

(b)

Figure 2. a) Lateral view of pyroelectric chamber; and b) Block diagram of the final circuit.

Table 2. Range of tube potential and the current of the X-ray equipment.

\begin{tabular}{cc}
\hline Peak tube potential $(\mathbf{k V p})$ & Fixed current $(\mathbf{m A})$ \\
\hline 120 & 20 \\
180 & 20 \\
200 & 20 \\
250 & 15 \\
300 & 12 \\
\hline
\end{tabular}

Cooper filter of $0.60,1.10,2.10$ and $4.20 \mathrm{~mm}$ were used for $180,200,250$ and $300 \mathrm{kVp}$, respectively. For $120 \mathrm{kVp}$ a $3 \mathrm{~mm}$ thick aluminum filter was used. Equivalent photon energy of the beam was determined from its measured halfvalue layer (HVL). The pyroelectric sensor was placed at $50 \mathrm{~cm}$ from the X-ray source, which is the commonly used distance in patients during treatment.

The energy fluency rate $(\Psi)$ of the beam was calculated from the measured $\mathrm{X}$-ray exposure rate $(\dot{\mathrm{X}})$ and air energy absorption coefficient $(\mu \mathrm{ab} / \rho)$ air using the Equation $2^{17}$ :

$\Psi=\dot{X} \frac{8.73 \times 10^{-3}}{\left(\mu_{a b} / \rho\right)_{a i r}}\left(\mathrm{~W} / \mathrm{m}^{2}\right)$

where $\dot{\mathrm{X}}$ was measured with a calibrated $0.6 \mathrm{~cm}^{3}$ cylindrical ionization chamber, connected to a FARMER model 2570 electrometer designed for clinical use in the orthovoltage range.

\section{Results and Discussion}

Pz34/PEEK composite film with 60 vol. $\%$ of ceramic was poled at $130{ }^{\circ} \mathrm{C}$ for 1 hours. By varying the applied electric field, the poling efficiency could be analyzed. To avoid breakdown, the polarization was carried out in a silicone oil bath. Figure 3 shows the longitudinal $d 33$ piezoelectric coefficient. An electric field up to $18 \mathrm{MV} / \mathrm{m}$ was applied without any damage to the sample and a piezoelectric constant of $29 \mathrm{pC} / \mathrm{N}$ was obtained.

On the pyroelectric side, the pre-poled sample with $15 \mathrm{MV} / \mathrm{m}$ was placed in a temperature-controlled chamber and the thermally stimulated discharge current (TSDC) was measured. The sample was heated at constant rate $\left(1^{\circ} \mathrm{C} / \mathrm{min}\right)$ with its electrodes short-circuited and the current was monitored with an electrometer (Keithley model 610C). The reversible pyroelectric current was established after removing all contributions to the irreversible TSDC current, which occurred after 3rd TSDC run.

The pyroelectric coefficient $\mathrm{p}(\mathrm{T})$ as a function of temperature was calculated using the Equation 3:

$$
p(T)=\frac{1}{A} \frac{I_{p}}{d T / d t}
$$

where $A$ is the electroded area, I $p$ is the pyroelectric current and $d T / d t$ the heating rate. Figure 4 shows the behavior of $\mathrm{p}(\mathrm{T})$ with the temperature. At room temperature the value of the pyroelectric coefficient is $19 \mu \mathrm{C} / \mathrm{m} 2 \mathrm{~K}$ and the pyroelectric figure of merit (FOM) is $0.43 \mu \mathrm{C} / \mathrm{m} 2 \mathrm{~K}$. No appreciable change in the piezo and pyroelectric activity was observed after the irradiation $(0.2 \mathrm{C} / \mathrm{kg} \approx 760$ Roentgens), indicating that there is no degradation in the sensing element in the energy range used.

To measure the absorbed energy by the sensor materials, a $7 \mathrm{~mm}$ thick lead foil with a $7 \mathrm{~mm}$ diameter hole was placed $50 \mathrm{~cm}$ from the X-ray tube. The exposure was measured with and without the sensors covering the hole. Table 3 shows the absorbed energy percentage of Pz34 ceramic, LiNbO3 crystal and Pz34/PEEK composite for all tube potential ranges.

It can be seen from the data in Table 3 that the radiation was not completely absorbed by the sensor materials. The thickness of the Pz34 ceramic and the LiNbO3 crystal were $1 \mathrm{~mm}$. 


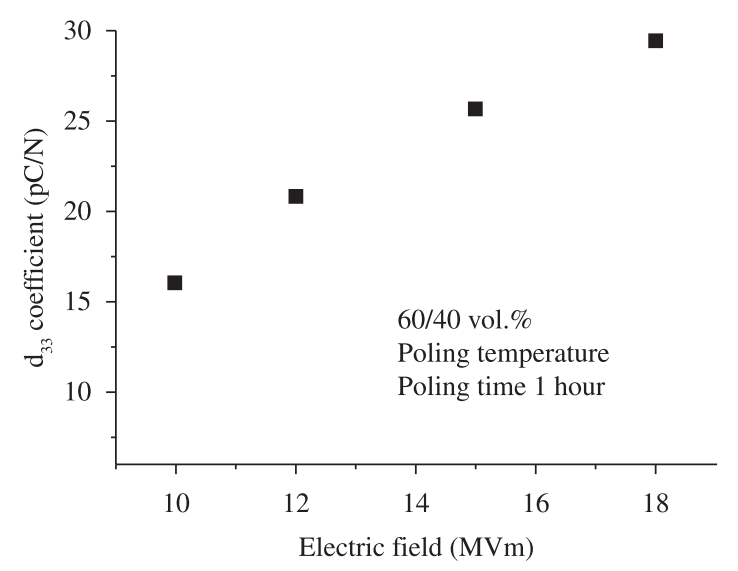

Figure 3. Longitudinal piezoelectric coefficient as a function of the poling electric field.

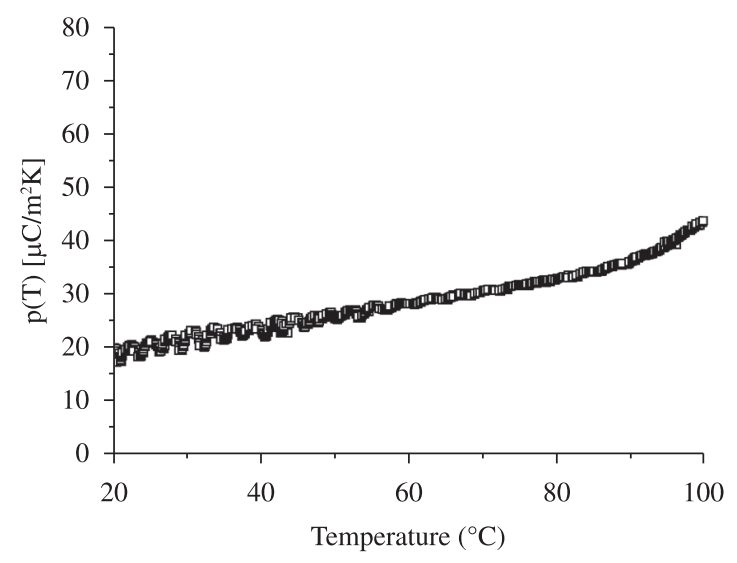

Figure 4. Pyroelectric coefficient for Pz34/PEEK 60/40 vol.\% poled with $15 \mathrm{MV} / \mathrm{m}$ electric field at $130{ }^{\circ} \mathrm{C}$.
Table 4 shows the half-value layer (HVL), energy of the photons, absorption coefficient of mass energy ( $\mu \mathrm{ab} / \rho$ ), exposure rate and the energy fluency rate $(\Phi)$, which are parameters for standard measure of radiation. The halfvalue layer is related with the interaction probability $(\mu)$ of the photon in the thickness unit of the material. The rate $\mu \mathrm{ab} / \rho$ is usually called cross section. The response of the pyroelectric sensor is proportional to the energy fluence rate or intensity.

The behavior of the sensor response as a function of energy fluency rate or X-ray intensity is shown in Figure 5. For comparison the responses of Pz34 ferroelectric ceramic and $\mathrm{LiNbO} 3$ crystal $1 \mathrm{~mm}$ thick are also plotted.

The electrical signal coming from the output of the high sensitive current-to-voltage converter is noise filtered and then fed into the microcontroller acquisition section. The microcontroller is interfaced through a serial port to receive commands from and send data to a computer. The X-ray intensity was calculated by dividing energy fluency by X-ray exposure time. The exposure was measured with a Radcal system model 9015 (Monrovia, CA, USA).

It can be seen from Figure 5 that for commonly energy used in radiation therapy $(120 \mathrm{kVP})$, the response of all sensing materials is higher. This behavior might be related with the energy absorption, which is 95.78, 83.91 and 79.78\% for Pz34, LiNbO3 and Pz34/PEEK, respectively. As the X-ray intensity increases, the absorption decreases and the sensor responses also decrease but the amplitude is sufficient to be accurately measured. The values presented in Figure 5 are the average values of 5 measurements and the repeatability was observed.

Using the composite material with just 60 vol.\% of ceramic, a pyroelectric system to measure X-ray intensity in the orthovoltage range was implemented. In spite of the lower radiation absorption by the composite in comparison with Pz34 ceramic or LiNbO3 crystal, the amplitude of its response is very similar.

Table 3. Energy absorption by the sensor material.

\begin{tabular}{ccccc}
\hline Material & $\begin{array}{c}\text { Tube potential } \\
(\mathbf{k V} \mathbf{p})\end{array}$ & $\begin{array}{c}\text { Exposure without sensor } \\
(\mathbf{R} / \mathbf{m i n})\end{array}$ & $\begin{array}{c}\text { Exposure with sensor } \\
(\mathbf{R} / \mathbf{m i n})\end{array}$ & $\begin{array}{c}\text { Absorption } \\
(\%)\end{array}$ \\
\hline LiNb03 & 120 & 7.58 & 1.22 & 83.91 \\
Pz34 & 120 & 7.58 & 0.32 & 95.78 \\
Pz34/PEEK & 120 & 7.58 & 1.56 & 79.42 \\
LiNb03 & 180 & 13.44 & 4.62 & 65.63 \\
Pz34 & 180 & 13.44 & 1.12 & 91.67 \\
Pz34/PEEK & 180 & 13.44 & 4.62 & 65.63 \\
LiNb03 & 200 & 10.06 & 5.00 & 50.30 \\
Pz34 & 200 & 10.06 & 1.34 & 86.68 \\
Pz34/PEEK & 200 & 10.06 & 4.50 & 55.27 \\
LiNb03 & 250 & 10.64 & 7.14 & 32.89 \\
Pz34 & 250 & 10.64 & 2.58 & 75.75 \\
Pz34/PEEK & 250 & 10.64 & 6.12 & 42.48 \\
LiNb03 & 300 & 4.84 & 4.02 & 16.94 \\
Pz34 & 300 & 4.84 & 1.92 & 60.33 \\
Pz34/PEEK & 300 & 4.84 & 3.48 & 28.10 \\
\hline
\end{tabular}


Table 4. Parameters for standard measure of radiation. $\mathrm{Al}$ and $\mathrm{Cu}$ are filters.

\begin{tabular}{cccccc}
\hline $\begin{array}{c}\text { Tube potential } \\
(\mathbf{k V p})\end{array}$ & $\begin{array}{c}\text { HVL } \\
(\mathbf{m m})\end{array}$ & $\begin{array}{c}\text { Energy of photon } \\
(\mathbf{k e V})\end{array}$ & $\begin{array}{c}\mu \mathbf{a b} / \rho \\
\left(\mathbf{m}^{\mathbf{2}} \mathbf{. k g} \mathbf{- 1}\right)\end{array}$ & $\begin{array}{c}\text { Exposure rate } \\
(\mathbf{R} / \mathbf{m i n})\end{array}$ & $\begin{array}{c}\Phi \\
\left(\mathbf{W} \cdot \mathbf{m}^{-\mathbf{2}}\right)\end{array}$ \\
\hline 120 & $3.00(\mathrm{Al})$ & 34.69 & 0.0626 & 45.20 & 6.30 \\
180 & $0.60(\mathrm{Cu})$ & 67.13 & 0.1030 & 82.50 & 6.99 \\
200 & $1.10(\mathrm{Cu})$ & 83.67 & 0.0511 & 64.10 & 10.95 \\
250 & $2.10(\mathrm{Cu})$ & 118.68 & 0.0223 & 66.80 & 26.10 \\
300 & $4.20(\mathrm{Cu})$ & 178.19 & 0.0078 & 33.10 & 37.19 \\
\hline
\end{tabular}

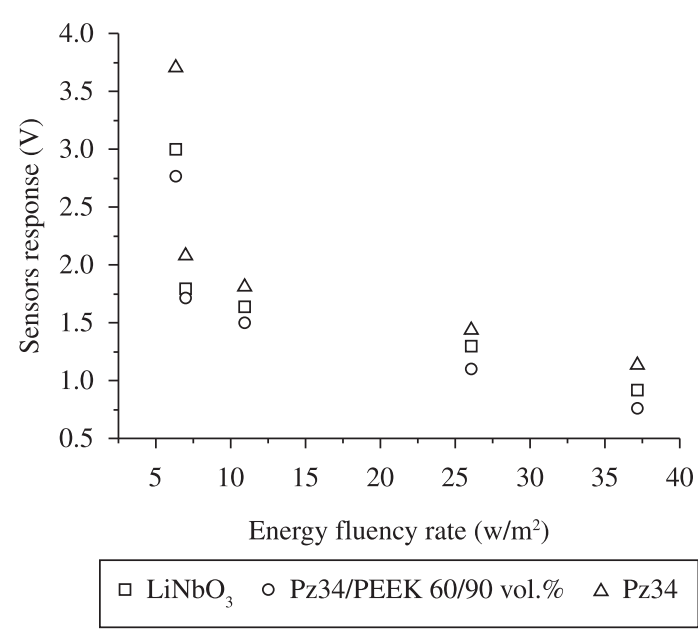

Figure 5. Sensors response to X-ray intensity in the orthovoltage range.

Although the relationship between the response and the energy fluency rate is not linear, there is no double value of voltage for different energies, indicating that the composite Pz34/PEEK can be used as sensing material for radiation detection. Research is in progress in our lab to show that the composite film can be also used for lower energy range and for non-ionizing radiation as infrared.

\section{Conclusions}

A new instrument using a pyroelectric composite made of Pz34 ceramic and PEEK polymer matrix was described

\section{References}

1. Hester DL, Glower DD and Overton LJ. Use of Ferroelectrics for Gamma-Ray Dosimetry. IEEE Transactions on Nuclear Science. 1964; 11:145-148. http://dx.doi.org/10.1109/ TNS2.1964.4315485

2. De Carvalho AA, Brassalotti AL, De Paula MH and Alter AJ. Use of lithium niobate detector for measuring X-ray intensity in mammographic range. Electronics Letters. 2004; 40:1097-1098. http://dx.doi.org/10.1049/el:20045729

3. Stadlober B, Zirkl M, Leising G, Gaar N, Bauer-Gogonea S and Bauer S. Transparent pyroelectric sensors and organic field-effect transistors with fluorinated polymers: steps towards organic infrared detectors. IEEE Transactions on Dielectrics and Electrical Insulation. 2006; 13(5):1087-1092. as an alternative system to an expensive ionization chamber for dosimetry. The instrument measures X-ray intensity in the orthovoltage range directly and can accurately monitor the application of radiation dose therapy. The response of the sensing element was not linear with the radiation intensity between 120 to $180 \mathrm{kVp}$ but it does not become a problem because the instrument is microcontrolled. A linear relationship can be observed from 180 to $300 \mathrm{kVp}$.

The experimental results show that no degradation was observed in the composite material after irradiation. The pyroelectric coefficient and the piezoelectric coefficient d33 remained the same after the sensor received a high exposure (about $0.2 \mathrm{C} / \mathrm{kg}=760$ Roentgens) of radiation in the orthovoltage range. In the energy fluency range of 6.30 to $37.19 \mathrm{~W} . \mathrm{m}^{-2}$, the sensor response varies from 2.70 to $0.80 \mathrm{~V}$ without double values of voltage for different energies, at least in the range used. Research is in progress to show that this composite material can be used also in the mammography $\mathrm{X}$-ray range and in non-ionizing radiation as infrared.

\section{Acknowledgements}

The authors Express their gratitude to Fundação de Amparo à Pesquisa do Estado de São Paulo - FAPESP. WKS thanks FAPESP and Conselho Nacional de Desenvolvimento Científico e Tecnológico - CNPq for financial support through Instituto Nacional de Ciência e Tecnologia de Materiais em Nanotecnologia - INCTMN.

4. Mokkonen-Craig S, Paronen M, Arstila K, Helariutta K, Ranhala E and Tikkanen P. Suitability of some common polymer films for $\mathrm{MeV}$ proton beam dosimetry. Nuclear Instruments and Methods in Physics Research Section B: Beam Interactions with Materials and Atoms. 2005; 236:366-370. http://dx.doi.org/10.1016/j.nimb.2005.03.278

5. Cameron JR, Kahn J and Hendee WR. A prospective study should be performed to test the hypothesis that an increase in background radiation to residents in the gulf states will increase their longevity. Medical Physics. 2002; 29(7):1511-1513. PMid:12148732. http://dx.doi.org/10.1118/1.1489045

6. Siebert BRL. Uncertainty in radiation dosimetry: basic concepts and methods. Radiation Protection Dosimetry. 2006; 121(1):3-11. PMid:16868011. http://dx.doi.org/10.1093/rpd/ncl094 
7. Metcalfe P, Kron T and Hoban P. The physics of radiotherapy $X$-rays: problems and solutions. Madison: Medical Physics Publishing; 1997. cap. 1-5.

8. Pacheco APL, Araújo ES and Azevedo WM. Polyaniline/poly acid acrylic thin film composites: a new gamma radiation detector. Materials Characterization. 2003; 50:245-248. http:// dx.doi.org/10.1016/S1044-5803(03)00090-1

9. Ryzhikov V, Gal'chinetskii L, Katrunov K, Lisetskaya E, Gavriluk V, Zelenskaya $\mathrm{O}$ et al. Composite detector for mixed radiations based on $\mathrm{CsI}(\mathrm{Tl})$ and dispersions of small $\mathrm{ZnSe}(\mathrm{Te})$ crystals. Nuclear Instruments and Methods in Physics Research Section A: Accelerators, Spectrometers, Detectors and Associated Equipment. 2005; 540:395-492. http://dx.doi. org/10.1016/j.nima.2004.12.030

10. De Paula MH, De Carvalho AA, Brassalotti AL, Alter AJ, Sakamoto WK, Malmonge JÁ et al. Microcontrolled pyro-electric instrument for measuring X-ray intensity in mammography. Medical and Biological Engineering and Computing. 2005; 43:751-755. http://dx.doi.org/10.1007/ BF02430953
11. Provisional Data Sheet PK1. Grades, properties and processing characteristics. Imperial Chemical Industries - ICI; 1980.

12. Charrier JM. Polymeric materials and processing. Munich: Hanser Publishers; 1991. p. 144-145.

13. Sakamoto WK. Espectroscopia dielétrica e corrente de despolarização termo-estimulada em filmes de peek. Eclética Química. 2003; 28(2):49-53. http://dx.doi.org/10.1590/S010046702003000200006

14. Pz34 Data Sheet. Available from: <http://www.ferropermpiezo.com>.

15. Byer RL and Roundy CB. Pyroelectric coefficient direct measurement technique and application to a nsec response time detector. Ferroelectrics. 1972; 3:333-338. http://dx.doi. org/10.1080/00150197208235326

16. Franco S. Design with operational amplifiers and analog integrated circuits, third ed.,WBC-MacGraw-Hill; 2002.

17. De Carvalho AA and Alter AJ. Measurement of X-ray intensity in the medical diagnostic range by a ferroelectric detector. IEEE Transactions on Ultrasonics, Ferroelectrics and Frequency Control. 1997; 44:1198-1203. http://dx.doi. org/10.1109/58.656620 